\title{
Measurement and evaluation of radar cross section for furniture in an indoor propagation channel
}

\author{
M. M. Maw ${ }^{1}$, P. Supanakoon ${ }^{1}$, S. Promwong ${ }^{1}$, and J. Takada ${ }^{2}$ \\ ${ }^{1}$ Faculty of Engineering, King Mongkut's Institute of Technology Ladkrabang, Chalongkrung Road, Ladkrabang, Bangkok \\ 10520, Thailand \\ ${ }^{2}$ Graduate School of Science and Engineering, Tokyo Institute of Technology, S6-4, 2-12-1, O-okayama, Meguro-ku, Tokyo \\ 152-8552, Japan
}

Correspondence to: M. M. Maw (myomyintmawphdit5@gmail.com)

Received: 9 January 2014 - Revised: 17 June 2014 - Accepted: 9 August 2014 - Published: 11 November 2014

\begin{abstract}
This paper has attempted to evaluate the radar cross section (RCS) of two furniture items in an indoor environment in a frequency range of $3-7 \mathrm{GHz}$ of the ultrawideband (UWB) range. The RCS evaluation is achieved through an extended version of the radar equation that incorporates the channel transfer function of scattering. The time-gating method was applied to remove the multipath effect, a phenomenon which typically occurs in the indoor environment. Two double-ridged waveguide horn antennas for both vertical and horizontal polarizations were used to obtain the transfer function of scattering of the furniture prior to analysis in order to derive their bistatic RCS. The RCS results validate the applicability of the proposed extended radar equation to the indoor propagation prediction.
\end{abstract}

\section{Introduction}

In an indoor environment, scattering targets, e.g., furniture items, walls, or even a human body, have an effect on the total received signal. In addition, the scatterers and their environment affect the radio wave propagation. Thus, the diverse effects of different scattering objects on the radio wave propagation warrant examination and analysis. In the case of furniture items, their multi-shape geometry necessitates special treatment to investigate and model their effects on the radio wave propagation.

Techniques have been proposed for the determination of the radar cross section (RCS) of various types of targets. Depending on the target size and selected frequency range, RCS can be investigated in either an outdoor environment, indoor environment, or inside an anechoic chamber. Miacci et al. (2012), Nicolaescu and Iubu (2007), and Bocanegra et al. (2008) examined the RCS of scattering objects in an anechoic chamber. Esposti et al. (2007) and Pongsilamanee and Bertoni (2004) analyzed the RCS of typical building walls, and Jong and Herben (2004) investigated the scattering of trees. Ghoraisti et al. (2009) studied the scattering from lampposts, traffic lights, and signboards. Tsuchiya et al. (2008) researched the Bragg scattering from periodic surfaces in an ultra-wideband (UWB) signal transmission. Lim et al. (2006) used the full wave simulation technique to investigate the scattering from multi-shape or porous objects, e.g., bricks, tables, and chairs. Kishiki and Takada (2008) studied the scattering of buildings in a street microcell environment, and Cheffena (2012) simulated the effect of a moving human body on the statistical channel.

This paper measures the channel transfer function of scattering from the furniture items in the indoor environment to obtain the magnitude and phase. The bistatic scattering measurements are analyzed by means of the extended radar equation and the Kaiser-Bessel window to derive the RCS of the furniture items. The organization of the paper is as follows: after this introduction, Sect. 2 details the underlying theory of RCS analysis in this paper. Section 3 deals with the experimental setup and procedure. Section 4 describes the RCS measurement results. The concluding remarks are provided in Sect. 5. 


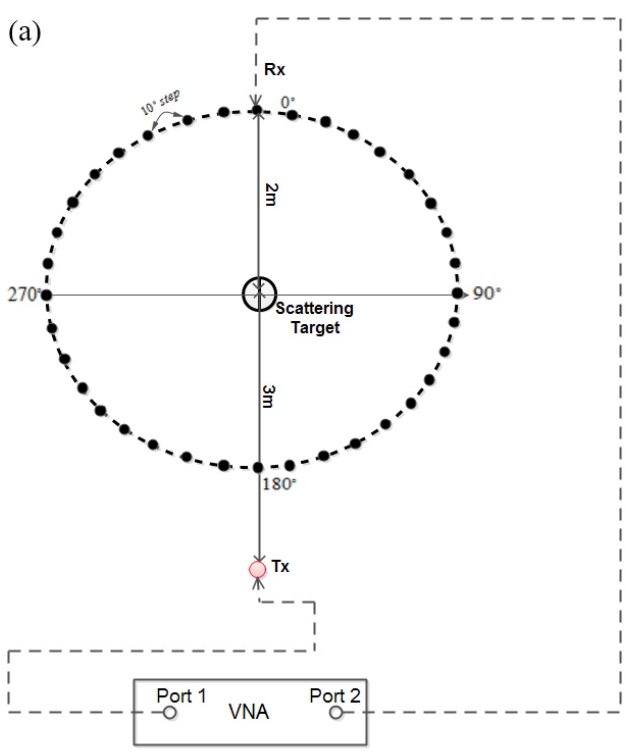

(b)

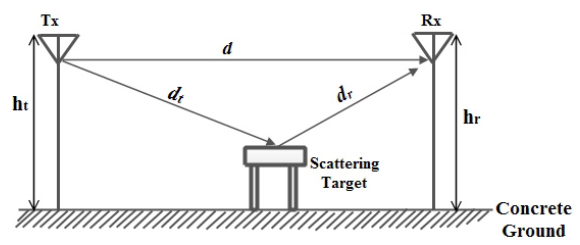

Figure 1. Top view and side view of the bistatic RCS.

\section{Theory of RCS evaluation}

The radar equation (Knott et al., 1985) is commonly used to describe the relationship between the received power and a target. For the radar link from a transmitter antenna (Tx) to a receiver antenna $(\mathrm{Rx})$, and with the polarizations of both antennas pointing to a scattering target, the received power $P_{\mathrm{r}}\left(f, \theta_{\mathrm{i}}, \varphi_{\mathrm{i}}, \theta_{\mathrm{s}}, \varphi_{\mathrm{s}}\right)$ can be calculated from

$$
\begin{aligned}
& P_{\mathrm{r}}\left(f, \theta_{\mathrm{i}}, \varphi_{\mathrm{i}}, \theta_{\mathrm{s}}, \varphi_{\mathrm{s}}\right) \\
& =\frac{c^{2}}{64 \pi^{3} f^{2} d_{\mathrm{t}}^{2} d_{\mathrm{r}}^{2}} \sigma\left(f, \theta_{\mathrm{i}}, \varphi_{\mathrm{i}}, \theta_{\mathrm{s}}, \varphi_{\mathrm{s}}\right) G_{\mathrm{t}}(f) G_{\mathrm{r}}(f) P_{\mathrm{t}}(f),
\end{aligned}
$$

where $P_{\mathrm{t}}(f)$ is the transmitted power, $G_{\mathrm{t}}(f)$ is the gain of $\mathrm{Tx}, G_{\mathrm{r}}(f)$ is the gain of $\mathrm{Rx}, d_{\mathrm{t}}$ and $d_{\mathrm{r}}$ are the respective distances from the target to Tx and $\mathrm{Rx}, \sigma\left(f, \theta_{\mathrm{i}}, \varphi_{\mathrm{i}}, \theta_{\mathrm{s}}, \varphi_{\mathrm{s}}\right)$ is the RCS of the target, $c$ is the velocity of light, $f$ is a carrier frequency, $\theta_{\mathrm{i}}$ and $\varphi_{\mathrm{i}}$ are the elevation and azimuth incident angles to the target, and $\theta_{\mathrm{S}}$ and $\varphi_{\mathrm{S}}$ are the elevation and azimuth scattering angles from the target.

This research work has adopted the extended version of the radar equation which incorporates the channel transfer function of scattering to evaluate RCS. The experimental targets are singly placed between Tx and Rx under a bistatic condition. The channel transfer function of scattering in frequency domain, $H_{\mathrm{sc}}\left(f, \theta_{\mathrm{i}}, \varphi_{\mathrm{i}}, \theta_{\mathrm{s}}, \varphi_{\mathrm{s}}\right)$, can be written as

$$
\begin{aligned}
& H_{\mathrm{sc}}\left(f, \theta_{\mathrm{i}}, \varphi_{\mathrm{i}}, \theta_{\mathrm{s}}, \varphi_{\mathrm{s}}\right) \\
& =\left[\frac{c}{8(\pi)^{3 / 2} f d_{\mathrm{t}} d_{\mathrm{r}}} H_{\sigma}\left(f, \theta_{\mathrm{i}}, \varphi_{\mathrm{i}}, \theta_{\mathrm{s}}, \varphi_{\mathrm{s}}\right) e^{-j 2 \pi f\left(d_{\mathrm{t}}+d_{\mathrm{r}}\right) / c}\right. \\
& \left.+H_{\mathrm{c}}\left(f, \theta_{\mathrm{i}}, \varphi_{\mathrm{i}}, \theta_{\mathrm{s}}, \varphi_{\mathrm{s}}\right)\right] H_{\mathrm{t}}(f) H_{\mathrm{r}}(f),
\end{aligned}
$$

where $H_{\mathrm{c}}\left(f, \theta_{\mathrm{i}}, \varphi_{\mathrm{i}}, \theta_{\mathrm{s}}, \varphi_{\mathrm{s}}\right)$ is the transfer function in the indoor environment without scattering targets, such as floor, walls, ceiling, and the mutual coupling between Tx and Rx; $H_{\mathrm{t}}(f)$ is the transfer function of $\mathrm{Tx} ; H_{\mathrm{r}}(f)$ is the transfer function of $\mathrm{Rx}$; and $H_{\sigma}\left(f, \theta_{\mathrm{i}}, \varphi_{\mathrm{i}}, \theta_{\mathrm{s}}, \varphi_{\mathrm{s}}\right)$ is the channel transfer function of scattering, where $\sigma$ can be determined from

$\sigma\left(f, \theta_{\mathrm{i}}, \varphi_{\mathrm{i}}, \theta_{\mathrm{s}}, \varphi_{\mathrm{s}}\right)=\left|H_{\sigma}\left(f, \theta_{\mathrm{i}}, \varphi_{\mathrm{i}}, \theta_{\mathrm{s}}, \varphi_{\mathrm{s}}\right)\right|^{2}$.

The experiments attempt to evaluate the indoor RCS of the furniture items, $H_{\sigma}\left(f, \varphi_{\mathrm{s}}\right)$. However, the channel transfer function in an indoor environment without scattering, $H_{\mathrm{c}}\left(f, \varphi_{\mathrm{s}}\right)$, is inevitable in such measurement. The timegating method is applied to remove $H_{\mathrm{c}}\left(f, \varphi_{\mathrm{s}}\right)$ using the Kaiser-Bessel window in time domain, $w\left(t, \theta_{\mathrm{i}}, \varphi_{\mathrm{i}}, \theta_{\mathrm{s}}, \varphi_{\mathrm{s}}\right)$ (Eq. 4), and the impulse response of scattering component, $h_{\mathrm{sc}}\left(t, \theta_{\mathrm{i}}, \varphi_{\mathrm{i}}, \theta_{\mathrm{s}}, \varphi_{\mathrm{s}}\right)$ (Eq. 6). Then, the channel transfer function after time gating, $H_{\mathrm{ti}}\left(f, \varphi_{\mathrm{s}}\right)$ (Eq. 7), is scaled by the channel transfer function in free space, $H_{\mathrm{fr}}(f)$ (Eq. 8), to remove $H_{\mathrm{t}}(f)$ and $H_{\mathrm{r}}(f)$. The final result is $H_{\sigma}\left(f, \varphi_{\mathrm{s}}\right)$ (Eq. 9).

The Kaiser-Bessel window is used in the time domain to extract scattering from the target at a delay time of $\left(d_{\mathrm{t}}+d_{\mathrm{r}}\right) / c$. The Kaiser-Bessel window $w\left(t, \theta_{\mathrm{i}}, \varphi_{\mathrm{i}}, \theta_{\mathrm{s}}, \varphi_{\mathrm{s}}\right)$ is defined as

$$
\begin{array}{cc}
\frac{w\left(t, \theta_{\mathrm{i}}, \varphi_{\mathrm{i}}, \theta_{\mathrm{s}}, \varphi_{\mathrm{s}}\right)=}{\mathrm{I}_{0}\left(\pi \alpha \sqrt{1-\left\{\frac{2\left[t-t_{\mathrm{p}}\left(\theta_{\mathrm{i}}, \varphi_{\mathrm{i}}, \theta_{\mathrm{s}}, \varphi_{\mathrm{s}}\right)\right]}{T}\right\}^{2}}\right)} & t_{\mathrm{p}}-\frac{T}{2} \leq t \leq t_{\mathrm{p}}+\frac{T}{2} \\
\mathrm{I}_{0}(\pi \alpha) & \text { otherwise, }
\end{array}
$$

where $T$ is the window width, $\alpha$ is a window shape parameter whose value is $4.8 ; t_{\mathrm{p}}\left(\theta_{\mathrm{i}}, \varphi_{\mathrm{i}}, \theta_{\mathrm{s}}, \varphi_{\mathrm{s}}\right)$ is set at the time around $\left(d_{\mathrm{t}}+d_{\mathrm{r}}\right) / c$ to capture the impulse response corresponding to the scattering component; and $\mathrm{I}_{0}(x)$ is the zeroth-order modified Bessel function of the first kind, which can be expressed as

$\mathrm{I}_{0}(x)=\frac{1}{\pi} \int_{0}^{\pi} e^{x \cos (\theta)} d \theta$.

The impulse response of the scattering component $h_{\mathrm{sc}}\left(t, \theta_{\mathrm{i}}, \varphi_{\mathrm{i}}, \theta_{\mathrm{s}}, \varphi_{\mathrm{s}}\right)$ can be calculated from

$$
h_{\mathrm{sc}}\left(t, \theta_{\mathrm{i}}, \varphi_{\mathrm{i}}, \theta_{\mathrm{s}}, \varphi_{\mathrm{s}}\right)=\int_{-\infty}^{\infty} H_{\mathrm{sc}}\left(f, \theta_{\mathrm{i}}, \varphi_{\mathrm{i}}, \theta_{\mathrm{s}}, \varphi_{\mathrm{s}}\right) e^{j 2 \pi f t} d f .
$$




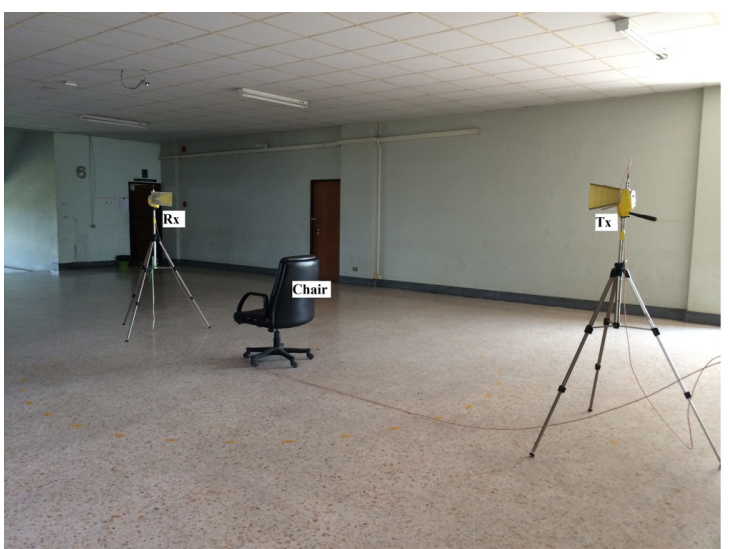

(a) VV polarization

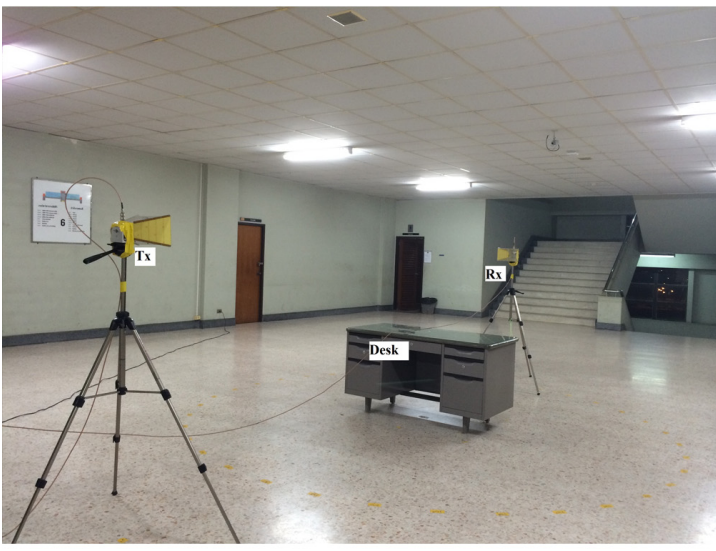

(c) VV polarization

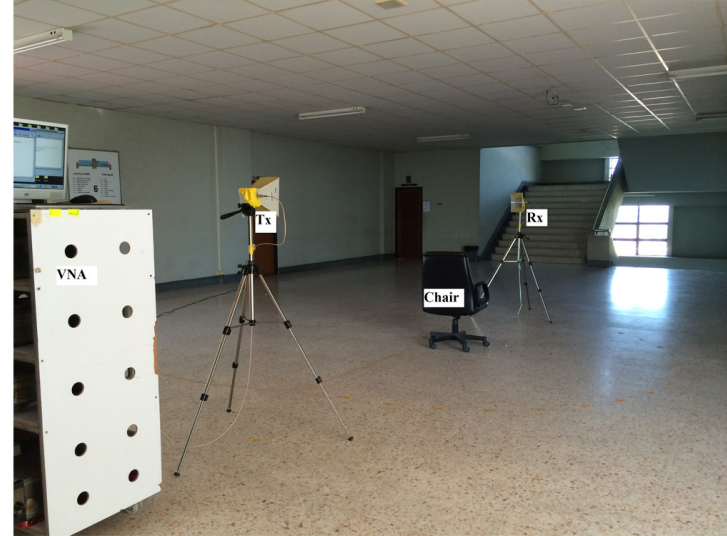

(b) $\mathrm{HH}$ polarization

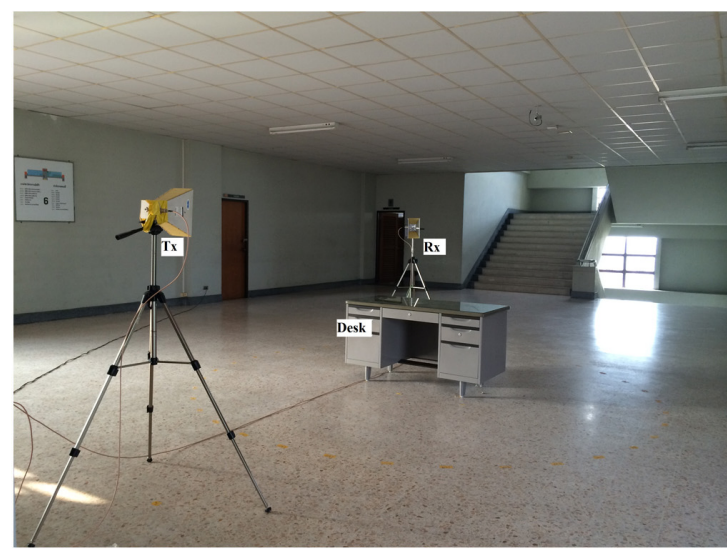

(d) HH polarization

Figure 2. Bistatic measurement setups for an office chair and a steel desk with VV and HH polarizations.

The frequency transfer function after time gating $H_{\mathrm{ti}}\left(f, \varphi_{\mathrm{s}}\right)$ can be estimated from

$$
\begin{aligned}
& H_{\mathrm{ti}}\left(f, \theta_{\mathrm{i}}, \varphi_{\mathrm{i}}, \theta_{\mathrm{s}}, \varphi_{\mathrm{s}}\right) \\
& =\int_{-\infty}^{\infty} h_{\mathrm{sc}}\left(t, \theta_{\mathrm{i}}, \varphi_{\mathrm{i}}, \theta_{\mathrm{s}}, \varphi_{\mathrm{s}}\right) w\left(t, \theta_{\mathrm{i}}, \varphi_{\mathrm{i}}, \theta_{\mathrm{s}}, \varphi_{\mathrm{s}}\right) e^{-j 2 \pi f t} d t \\
& \approx \frac{c}{8(\pi)^{3 / 2} f d_{\mathrm{t}} d_{\mathrm{r}}} H_{\sigma}\left(f, \varphi_{\mathrm{s}}\right) e^{-j 2 \pi f\left(d_{\mathrm{t}}+d_{\mathrm{r}}\right) / c} H_{\mathrm{t}}(f) H_{\mathrm{r}}(f)
\end{aligned}
$$

The measurement of the channel transfer function of free space, $H_{\mathrm{fr}}(f)$, was carried out in an anechoic chamber prior to substituting in Eq. (9) to remove $H_{\mathrm{t}}(f)$ and $H_{\mathrm{r}}(f)$. The Tx and Rx antennas were aligned such that they pointed at each other to obtain a direct link. The channel transfer function of free space, $H_{\mathrm{fr}}(f)$, can be written as

$$
H_{\mathrm{fr}}(f)=\frac{c}{4 \pi f d_{\mathrm{f}}} e^{-j 2 \pi f d_{\mathrm{f}} / c} H_{\mathrm{t}}(f) H_{\mathrm{r}}(f),
$$

where $d_{\mathrm{f}}$ is the distance from Tx to Rx.
The channel transfer function of scattering, or RCS, is finally derived and can be expressed as

$$
H_{\sigma}\left(f, \varphi_{\mathrm{s}}\right)=\frac{\sqrt{4 \pi} d_{\mathrm{t}} d_{\mathrm{r}}}{d_{\mathrm{f}}} e^{j 2 \pi f\left(d_{\mathrm{t}}+d_{\mathrm{r}}-d_{\mathrm{f}}\right) / c} \frac{H_{\mathrm{ti}}\left(f, \theta_{\mathrm{i}}, \varphi_{\mathrm{i}}, \theta_{\mathrm{s}}, \varphi_{\mathrm{s}}\right)}{H_{\mathrm{fr}}(f)} .
$$

\section{Experimental evaluation of sample furniture}

The location where the experiments were carried out was an indoor open area on the sixth floor of the E-Building, Faculty of Engineering, King Mongkut's Institute of Technology Ladkrabang. The measurements were taken on weekends to avoid interferences from the human body. A sequence of measurements were completely undertaken prior to analysis of the RCS of the experimental furniture items. The measurement setup and procedure are detailed next.

A vector network analyzer (VNA) (HP 8510C) was utilized to measure the magnitude and phase of the transmission coefficients. The VNA was operated in the response measurement mode, where port 1 and port 2 were the transmitter 
(a)

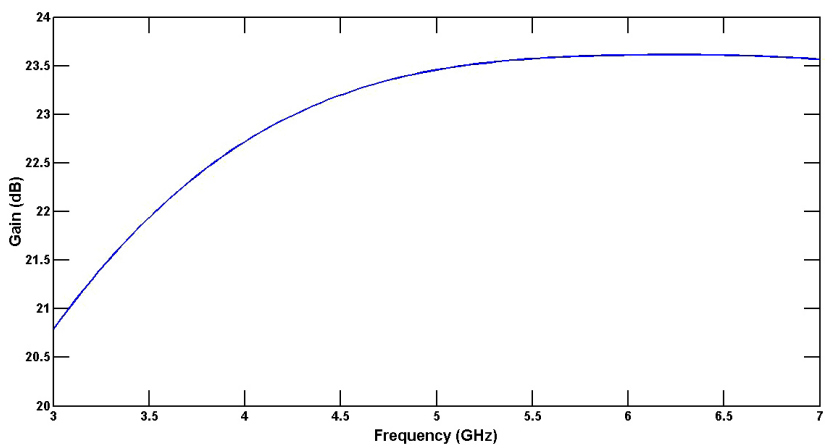

(b)

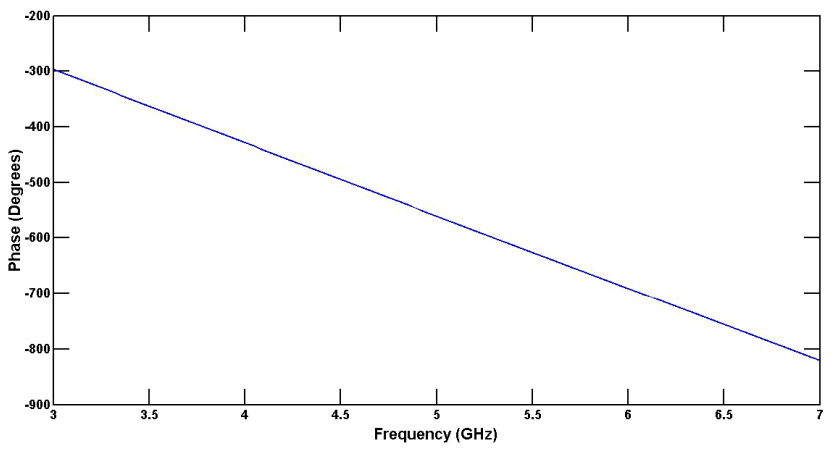

Figure 3. Gain and phase of horn antennas relative to frequency.

and receiver ports, respectively. The full two-port method was utilized to calibrate the analyzer to suppress the noise level and to improve the sensitivity and accuracy measurement. Two double-ridged waveguide horn antennas were used, one as Tx and the other as Rx, with an operating frequency range of $1-18 \mathrm{GHz}$ for both vertical and horizontal polarizations. Two experimental scattering targets were an office chair of $1 \mathrm{~m} \times 0.62 \mathrm{~m} \times 0.44 \mathrm{~m}(H \times W \times L)$ and a steel desk of $0.76 \mathrm{~m} \times 1.37 \mathrm{~m} \times 0.66 \mathrm{~m}(H \times W \times L)$.

In general, an accurate RCS is achievable only in an anechoic chamber in which the effects of walls, floor, ceiling, and other background clutters are removed. Nevertheless, since the available anechoic chamber is too small to fit the two horn antennas and either of the scattering targets with sufficient space remaining for running the experiments, the experiments were thus carried out in two steps: (1) the evaluation of RCS with the existence of either target in the indoor open area on the sixth floor of the E-Building, and (2) the antenna calibration in the anechoic chamber.

For the setup, the frequency range is from 3 to $7 \mathrm{GHz}$ with a total of 801 frequency points. The averaging factor was set at 4096 to reduce the noise level and improve the signal-to-noise ratio (SNR). The sweep time was $200 \mathrm{~ms}$ and the intermediate frequency bandwidth (IFBW) of the vector network analyzer was $10 \mathrm{kHz}$. The measurement at each angle required approximately $15 \mathrm{~min}$. The maximum transmitted power of $10 \mathrm{dBm}$ was selected to obtain the maximum

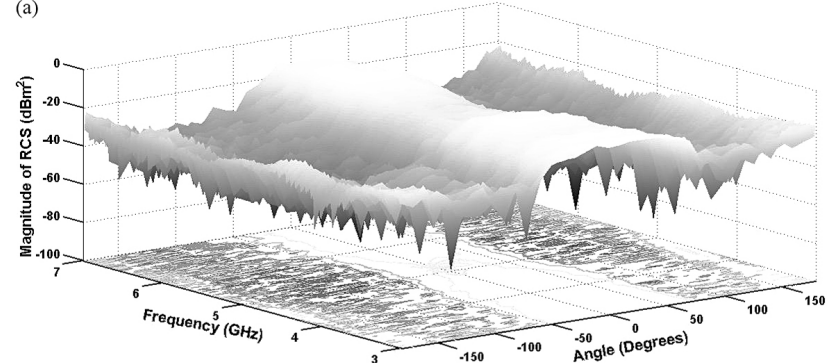

(b)

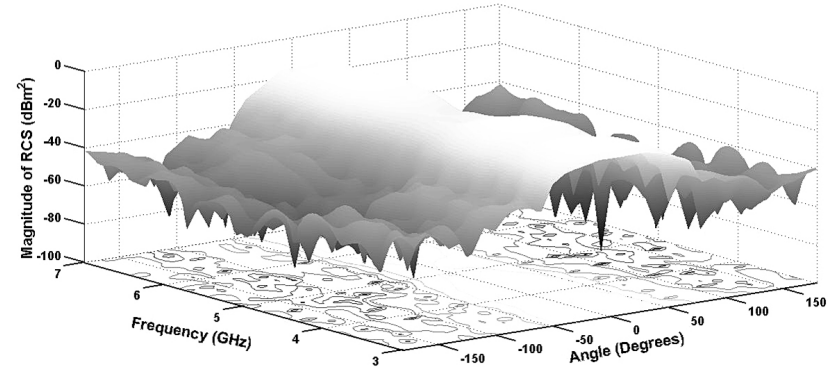

Figure 4. Comparison of RCS without and with time gating.

system dynamic range, and the received impulse response of each receiver position was recorded. For the RCS in the presence of either of the scattering targets, the distance from Tx to the center of the target was $3 \mathrm{~m}$, and the distance between the center of the target and the Rx antenna was $2 \mathrm{~m}$. The experiments were conducted in the near-field (Fresnel) region due to space and power limitations. Both Tx and Rx were placed at a height of $1.5 \mathrm{~m}$ from the ground. The receiving antenna was rotated with a $10^{\circ}$ increment around the scattering target for a total of 36 positions. The measurements were thus repeated by varying the angle of $\mathrm{Rx}$, while that of Tx remained stationary to achieve the directional pattern of the bistatic RCS between Tx and Rx, assuming that $\theta_{\mathrm{i}}=\theta_{\mathrm{s}}=90^{\circ}, \varphi_{\mathrm{i}}=180^{\circ}$, and $\sigma\left(f, \theta_{\mathrm{i}}, \varphi_{\mathrm{i}}, \theta_{\mathrm{s}}, \varphi_{\mathrm{s}}\right)$ is simplified to $\sigma\left(f, \theta_{\mathrm{s}}\right)$. Figure 1 illustrates the top and side views of the bistatic RCS, while Fig. 2a-d are photographs of the measurement setups for the chair and desk for vertical-vertical (VV) and horizontal-horizontal (HH) polarizations.

In the calibration, the antenna measurement in the anechoic chamber was carried out to determine the channel transfer function of free space. The Tx and Rx antennas in the calibration were each $1.40 \mathrm{~m}$ in height from the ground and were placed at a distance of $2 \mathrm{~m}$ and aligned pointing at each other. Figure 3 shows the broadside-broadside $\left(0^{\circ}\right)$ gains and phases of the horn antennas relative to the frequency.

\section{Experimental results}

This section presents how the accuracy of the conventional ray tracing simulation technique can be improved upon with the proposed extended radar equation. The indoor RCS of 


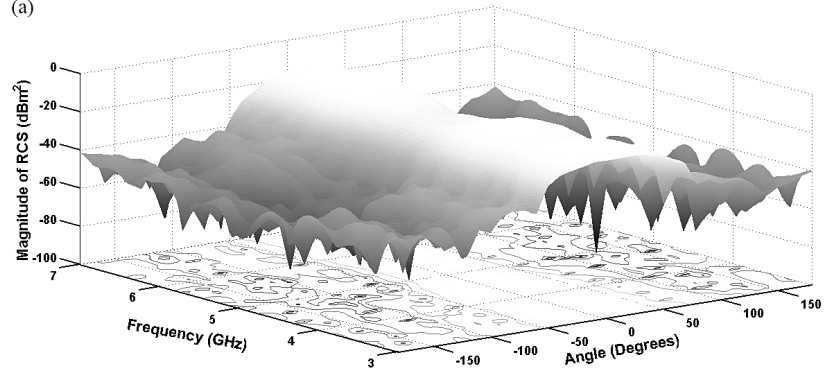

(b)

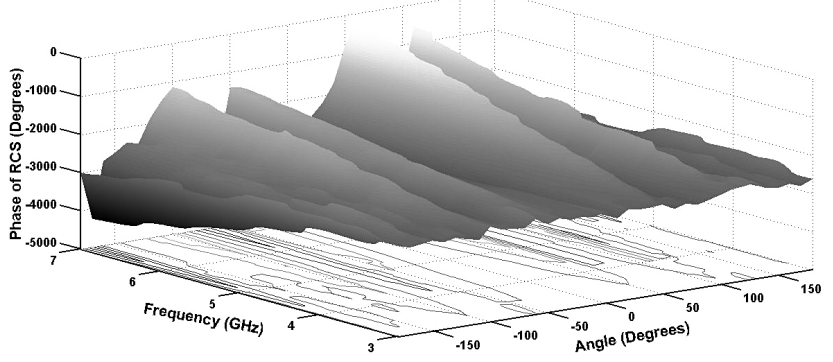

Figure 5. The RCS for the experimental office chair for VV polarization.

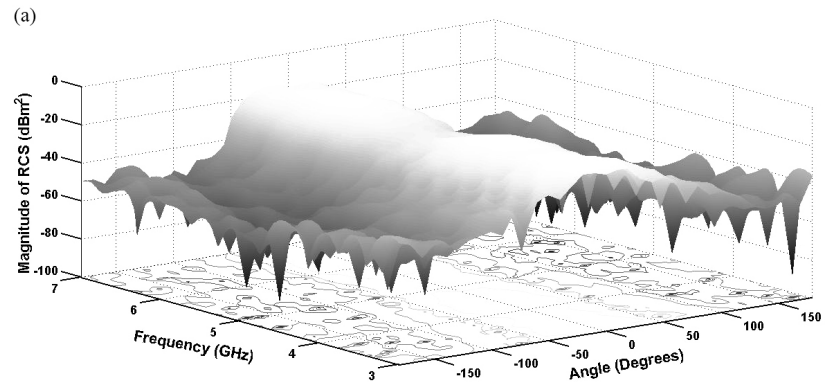

(b)

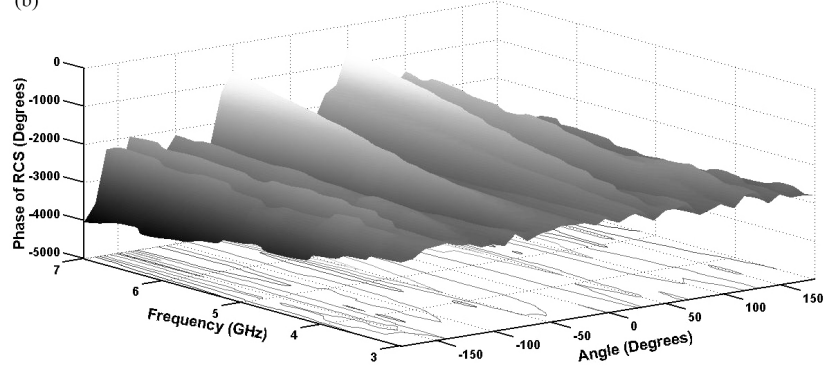

Figure 6. The RCS for the experimental office chair for $\mathrm{HH}$ polarization.

the two scattering targets, i.e., the office chair and steel desk, were evaluated in the frequency range of $3-7 \mathrm{GHz}$ in $\mathrm{VV}$ polarization and $\mathrm{HH}$ polarization. It is found that the $\mathrm{VV}$ and $\mathrm{HH}$ polarizations yielded different RCS.

This paper utilized time gating to remove the multipath effect in the received signals. The time-gating method transforms the frequency domain response into a time domain re-
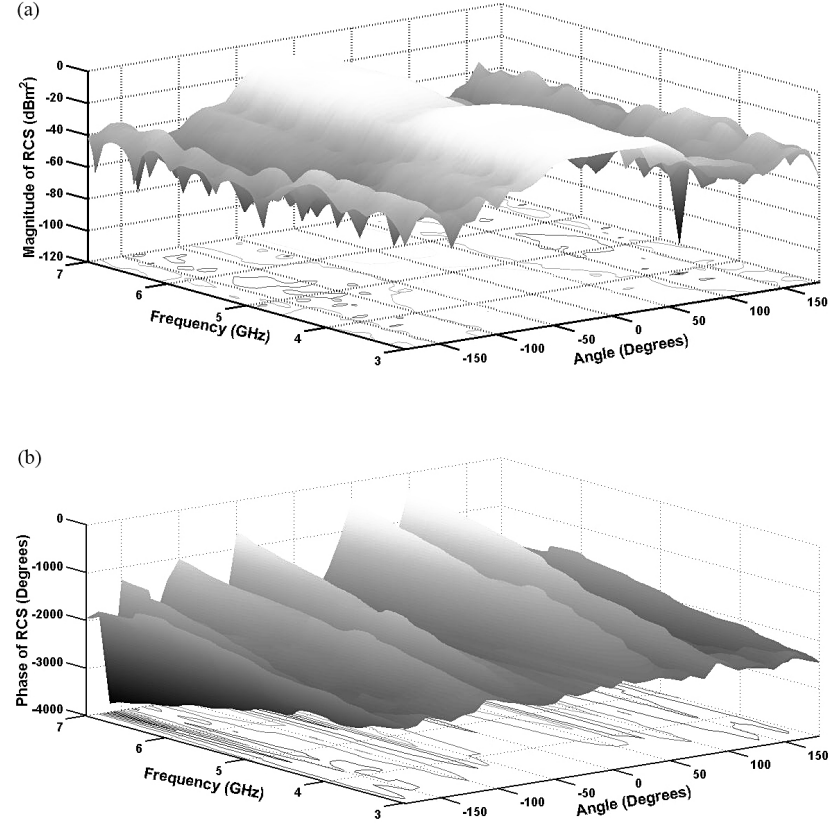

Figure 7. The RCS for the experimental steel desk for VV polarization.
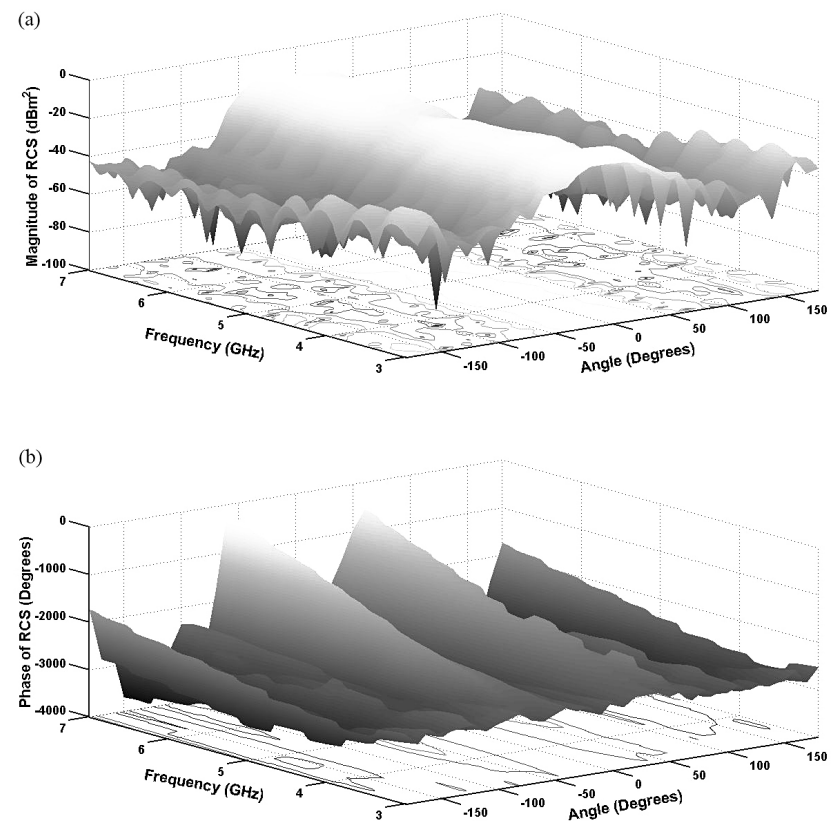

Figure 8. The RCS for the experimental steel desk for HH polarization.

sponse and also filters out the late time pulses, which are attributable to the multipath effect. To demonstrate the effectiveness of time gating in removing the multipath effect, the comparison between the RCS of the chair without and with time gating is provided. Figure 4 compares the RCS without and with time gating along the frequency range of $3-7 \mathrm{GHz}$ and with different incident angles. In Fig. 4a, without time 
gating, the scattering from the chair induces the interferences and ripples in the RCS, while in Fig. $4 \mathrm{~b}$, the time gating reduces the ripples in the main beam. Moreover, the scattering lobes beyond $90^{\circ}$ are reduced to below $-20 \mathrm{dBm}^{2}$, resulting in a significant reduction in the edge diffraction effect, which in turn makes the scattering effect easier to extract. As shown in Fig. 4b, the multipath effects from 90 to $180^{\circ}$ and -90 to $-180^{\circ}$ along the frequency range of $3-7 \mathrm{GHz}$ are considerably removed. With time gating, more accurate RCS can be achieved.

The 3-D graphics of RCS with time gating of the experimental chair for $\mathrm{VV}$ and $\mathrm{HH}$ polarizations are illustrated in Figs. 5 and 6, respectively, while those of the desk for VV and $\mathrm{HH}$ polarizations are depicted in Figs. 7 and 8. It was observed that the RCS for $\mathrm{HH}$ polarization is larger than that of $\mathrm{VV}$ polarization for most observation angles. In addition, the RCS varies with changes in incident angle and frequency. For instance, the RCS at $0^{\circ}$ of the chair for VV polarization at the frequencies of 3 and $5 \mathrm{GHz}$ are -40 and $-62 \mathrm{dBm}^{2}$, respectively; for $\mathrm{HH}$ polarization they are -36 and $-53 \mathrm{dBm}^{2}$ at the same frequencies. In the case of the desk at $0^{\circ}$, the RCS for VV polarization at 3 and $5 \mathrm{GHz}$ are respectively -55 and $-47 \mathrm{dBm}^{2}$, and they are -36 and $-46 \mathrm{dBm}^{2}$ at the same frequencies for $\mathrm{HH}$ polarization. The findings indicate that the RCS is subject to types of scattering objects, angle, frequency, and polarization.

\section{Conclusions}

This paper has attempted to evaluate the RCS of two furniture items, i.e., an office chair and a steel desk, using the extended radar equation at the frequency range of 3-7 GHz. Time gating was utilized to remove the multipath effect, and its removal effectiveness is verified by the comparison between the RCS without and with time-gating method. This research paper has also investigated the scattering characteristics of the two furniture items for different frequencies, angles, and polarizations. The maximum RCS of the experimental office chair and steel desk are approximately -11 and $-9 \mathrm{dBm}^{2}$, respectively. In both experimental scattering objects, the VV-polarization RCS is generally lower than that for $\mathrm{HH}$ polarization. This is possibly due to the diffraction at the side edge.
Acknowledgements. The authors gratefully acknowledge the anonymous reviewers and referees for their useful comments and constructive suggestions. The authors would like to extend deep gratitude to the Japan International Cooperation Agency (JICA) under the AUN/SEED-Net (ASEAN University Network, Southeast Asia Engineering Education Development Network) program. Sincere appreciation is also extended to the Wireless Communication Laboratory for the support with regard to the anechoic chamber. Special credit goes to S. Teawchim, S. Duanhsuwan, J. Sahakit, and W. Vinicchayakul for their assistance and contributions to this research.

Edited by: M. Chandra

Reviewed by: G. Wanielik and one anonymous referee

\section{References}

Bocanegra, D. E., Martinez, D. P., Recio., R. F., Lucena, A. J., and Sanchez, I. M.: New Benchmark Radar Targets for Scattering Analysis and Electromagnetic Software Validation, Prog. Electromagn. Res., 88, 39-52, 2008.

Cheffena, M.: Physical-Statistical Channel Model for Signal Effect by Moving Human Bodies, EURASIP J. Wirel. Comm., 77, 113, 2012.

Esposti, V. D., Fuschini, F., Vitucci, E. M., and Falciasecca, G.: Measurement and Modelling of Scattering from Buildings, IEEE T. Antenn. Propag., 55, 143-153, 2007.

Ghoraisti, M., Takada, J.-I., and Imai, T.: Radio Wave Scattering from Lamposts in Microcell Urban Mobile Propagation Channel, ECTI-EEC, 7, 14-20, 2009.

Jong, Y. L. C. and Herben, M. H. A. J.: A Tree-Scattering Model for Improved Propagation Prediction in Urban Microcells, IEEE T. Veh. Technol., 2, 503-513, 2004.

Kishiki, Y., and Takada, J.-I.: Improvement of 3D ray tracing simulation in microcell environment by introducing the complex radar cross section, The 2008 International Symposium on Antennas and Propagation (ISAP 2008), Taipei, Taiwan, 27-30 October 2008, 790-793, 2008.

Knott, E. F., Shaeffer, J. F., and Tuley, M. T.: Radar Cross Section, Artech House, New Jersey, 1985.

Lim, C.-P., Volakis, J. L., Sertel, K., Kindt, R. W., and Anastasopoulo, A.: Indoor Propagation models based on rigorous methods for site-specific multipath environment, IEEE T. Antenn. Prop., 54, 1718-1725, 2006.

Miacci, M. A. S., Nohara, E. L., Martin, I. M., Peixoto, G. G., and Rezende, M. C.: Indoor Radar Cross Section Measurement of Simple Targets, Journal of Aerospace Technology and Management (JATM), 4, 25-32, 2012.

Nicolaescu, I. and Iubu, G.: Simple and Collected Targets Radar Cross Section, International Conference on Electromagnetics in Advanced Applications (ICEAA 2007), Torino, 17-21 September 2007, 295-298, 2007.

Pongsilamanee, P. and Bertoni, H. L.: Specular and Nonspecular Scattering from Building Facades, IEEE T. Antenn. Prop., 52, 1879-1889, 2004.

Tsuchiya, H., Lertsirisopon, N., Takada, J.-I., and Kobayashi, T.: Effects of Bragg Scattering on Ultra-Wideband Signal Transmission from Periodic Surfaces, IEICE Transactions on Communications, E91-B, 536-542, 2008. 